\title{
Facilitative Effects of the Ampakine CX516 on Short-Term Memory in Rats: Enhancement of Delayed-Nonmatch-to-Sample Performance
}

\author{
Robert E. Hampson, ${ }^{1}$ Gary Rogers, ${ }^{2}$ Gary Lynch, ${ }^{3}$ and Sam A. Deadwyler ${ }^{1}$ \\ ${ }^{1}$ Department of Physiology and Pharmacology, Wake Forest University School of Medicine, Winston Salem, North \\ Carolina 27157, ${ }^{2}$ Cortex Pharmaceuticals, Irvine, California 92718, and ${ }^{3}$ Department of Psychiatry, University of \\ California, Irvine, California 92715
}

\begin{abstract}
Ampakines are a family of drugs that selectively increase AMPA receptor-gated currents and improve performance on several behavioral tasks. This report describes evidence that ampakines cause a cumulative enhancement of performance in a spatial short-term memory task (Deadwyler et al., 1996). Two groups of rats were trained on a spatial variant of the delayednonmatch-to-sample (DNMS) paradigm. One group $(n=12)$ received the ampakine CX516 (Cortex Pharmaceuticals) alternated with vehicle for 17 consecutive days and then only vehicle for an additional $7 \mathrm{~d}$. The second group $(n=6)$ received only vehicle injections over the same number of days. CX516 improved performance within sessions, particularly on trials with delays of 6-35 sec. In 9 of 12 rats, the positive effect of the drug was also present on nondrug days between CX516 ad-
\end{abstract}

Drugs that positively modulate glutamatergic AMPA receptorgated currents increase the size of fast, excitatory synaptic responses (Ito et al., 1990; Issacson and Nicoll, 1991; Vyklicky et al., 1991) and reduce the amount of afferent activity needed to induce long-term potentiation (Staubli et al., 1994a). Either effect could promote the encoding of memory by facilitating the processing of cues and the formation of responses in circumstances involving familiar environments and practiced behaviors. Studies using hippocampal slices indicate that positive modulators of AMPA receptors have a much greater enhancing effect on polysynaptic than on monosynaptic responses (Arai et al., 1995). Accordingly, the modulators are likely to have a pronounced influence on those behaviors subserved by exceedingly complex networks running through hippocampus and cortex.

Several studies using ampakines have tested, with favorable results, the prediction that positive AMPA receptor modulators enhance memory (Staubli et al., 1994b). Ampakines are a group of small benzamide compounds that slow the deactivation rate of AMPA receptors and increase the size and duration of excitatory responses in hippocampal slices (Arai and Lynch, 1992). The drugs readily cross the blood-brain barrier with expected results on synaptic potentials and LTP induction in freely moving rats. Ampakines improve retention scores in

\footnotetext{
Received Oct. 17, 1997; revised Jan. 15, 1998; accepted Jan. 16, 1998.

This work was supported by National Institute on Drug Abuse Grants DA03502 and DA00119 to S.A.D. and DA08549 to R.E.H., and by Cortex Pharmaceuticals. We thank Douglas R. Byrd, Joanne K. Konstantopoulos, and Janet R. Brooks for technical assistance.

Correspondence should be addressed to Sam A. Deadwyler, Department of Physiology and Pharmacology, Wake Forest University School of Medicine, Medical Center Boulevard, Winston Salem, NC 27157-1083.

Copyright (C) 1998 Society for Neuroscience $\quad 0270-6474 / 98 / 182740-08 \$ 05.00 / 0$
}

ministration and after cessation of CX516 injections. The animals that received only vehicle injections showed no improvement in DNMS performance over the entire $32 \mathrm{~d}$ of testing. Three of the 12 animals given CX516 did not exhibit "carryover" effects of the drug to the intervening (vehicle only) test sessions, but nonetheless exhibited superior performance during the first half of the session on days in which the ampakine was administered. Evaluation of errors suggests that the ampakine eliminated the necessity for a shift in response strategy that produced proactive interference on the following trial. Hippocampal involvement in these ampakine effects is discussed as a prelude to the second article in the series (Hampson et al., 1998).

Key words: ampakine; hippocampus; learning; memory; behavior; delay-dependent memory; proactive interference

radial mazes (Staubli et al., 1994b), facilitate the acquisition of a conditioned response (Shors et al., 1994), and reduce the number of trials needed to form stable olfactory memory (Larson et al., 1995), at dosages predicted to be effective from in vitro physiological experiments. Evidence has also shown that ampakines have a positive effect on the delayed recall of nonsense syllables as well as on several commonplace forms of memory in humans (Lynch et al., 1996).

The facilitatory effect of AMPA receptor modulators on the induction of long-term potentiation (LTP) is presumably attributable to a greater net depolarizing response to highfrequency stimulation (via enhanced AMPA receptor currents) and thus a greater likelihood of producing an NMDA receptor-mediated current of magnitude sufficient to trigger the formation of LTP. Given the evidence linking LTP to several varieties of memory (Morris et al., 1986; Larson and Lynch, 1988; Staubli et al., 1994b), it can be predicted that positive modulators of AMPA receptors should enhance, via effects on LTP, the encoding of new information across a number of paradigms.

The initial objective of the present study was to determine whether ampakines produce positive effects on hippocampaldependent memory. Rats were tested on a spatial, delayednonmatch-to-sample (DNMS) task, in which performance typically drops to random levels with 1 min of delay between the Sample and Recognition phases on the task. In this particular paradigm, the sources of DNMS errors have been analyzed in detail (Deadwyler et al., 1996; Hampson and Deadwyler, 1996a,b) and thus could be used to significantly extend the understanding of aspects of stimulus encoding and information retrieval that are influenced by ampakines. A second goal was to test for cumulative 
effects of ampakines to determine whether repeated exposure to AMPA receptor-gated modulation of synaptic currents promoted persistent changes in behavior. Because it allows for detailed and quantitative analyses of complex behavior over time, the DNMS paradigm was well suited for studying such changes.

A final reason for testing whether ampakines modify DNMS performance was that a positive result would open the way for studies of how the drugs influence neuronal activity in circuits essential to memory processes. Classic spatial memory delay-type tasks in rodents, including this one, are disrupted by removal of hippocampus (Dunnett, 1989; Hampson et al., 1995). Moreover, recent studies have indicated that patterns of cell firing in hippocampus at various phases of the task are predictive of performance within a trial (Deadwyler et al., 1996).

The results reported here indicate that the ampakine CX516, a drug that facilitates memory in aged rats and humans (Granger et al., 1996; Lynch et al., 1996), is a potent enhancer of short-term memory as measured in this form of DNMS task. CX516-treated animals showed not only marked enhancement of DNMS performance between sessions, but also exhibited a residual improvement on days after injection sessions. This article will focus on the behavioral changes produced by CX516 with respect to alterations in short-term spatial memory and strategies that control that performance and will provide a foundation for subsequent presentation of electrophysiological correlates of these effects in the companion article (Hampson et al., 1998) that follows.

\section{MATERIALS AND METHODS}

Subjects. Eighteen male Long-Evans rats ranging in age from 200 to $250 \mathrm{~d}$ were used as subjects. Several pilot studies with different doses and drugs were used before we decided on the parameters for dosage, injection time, and vehicle reported here and in the companion article. The study itself took $>2$ years and was run as three complete replications with approximately one third of the animals (4-7 per group) in each replication. All animals were trained to the same behavioral criteria before surgery was performed and retrained to that criteria after surgery and before testing was begun.

Surgery. As animals reached behavioral performance criterion on the DNMS task, they were surgically implanted with a multi-neuron recording device that consisted of a 16-electrode array aimed at the CA1 and CA3 subfields of the hippocampus (Deadwyler et al., 1996) (also see companion article, Hampson et al., 1998). Surgery was performed under ketamine $(100 \mathrm{mg} / \mathrm{kg})$ and xylazine $(10 \mathrm{mg} / \mathrm{kg})$ anesthesia. After surgery, the cranium was sealed with bone wax and dental cement, and the animal was allowed to recover its preoperative weight (usually 1 week). The scalp wound was treated periodically with Neosporin antibiotic, and animals were given an injection of Crysticillin (penicillin G, 300,000 U) to prevent infection. All animal care and experimental procedures conformed to National Institutes of Health and Society for Neuroscience guidelines for care and use of experimental animals.

Apparatus. The apparatus was similar to that used in other studies from this laboratory (Hampson et al., 1993; Deadwyler et al., 1996). Briefly, studies were conducted in $43 \times 43 \times 53 \mathrm{~cm}$ Plexiglas behavioral testing chambers (Eichenbaum et al., 1987) with manipulanda and other features similar to that initially described and modified by Hampson et al. (1993). The entire apparatus was housed inside a commercial sound-attenuated cubicle (Industrial Acoustics, Bronx, NY). On one wall of the chamber, two retractable levers (Coulborn Instruments, Lehigh Valley, PA) were positioned $3.5 \mathrm{~cm}$ above the floor and separated by $14.0 \mathrm{~cm}$, center to center. A water dispenser trough was positioned midway between the levers. The nosepoke device, consisting of an infrared photodetector and light-emitting diode spanning a $2.5 \mathrm{~cm} \times 1 \mathrm{~cm} \times 1 \mathrm{~cm}$ opening in an aluminum housing, was mounted $4.0 \mathrm{~cm}$ above the chamber floor, centered on the wall opposite the levers. The cue light $(6 \mathrm{~V}, 10 \mathrm{~W})$ was positioned immediately above the nosepoke device, and a speaker mounted overhead provided a constant $85 \mathrm{~dB}$ "white noise" background. Two $12 \mathrm{~V}, 25 \mathrm{~W}$ incandescent lamps (house lights) were mounted on the top of the chamber. Video monitoring of the animal at all times was provided by a Sanyo CCD black and white video camera mounted above the chamber. The apparatus was controlled by a minicomputer that collected all behavioral data and stored it on magnetic disks.

Behavioral training procedure. Animals were water-deprived but allowed free access to food for maintenance at $85-90 \%$ of their weight throughout the duration of DNMS training and recording. Periodically (every 30-60 d) animals were given free access to water and food, and a new weight was calculated to allow for normal body growth. Animals received water daily after the behavioral session. The water was typically consumed within $1 \mathrm{hr}$; therefore, before each successive behavioral session, all animals were typically water-deprived for $20-22 \mathrm{hr}$. The DNMS task was identical to that described by Deadwyler et al. (1996).

Pretraining was as described in Hampson et al. (1993). The task consisted of three main phases: Sample, Delay, and Recognition. At the initiation of a trial (Sample phase), either the left or right lever was extended (Sample presentation or SP), and the animal responded. This lever press constituted the Sample response or SR. The lever was retracted immediately, and the Delay phase was initiated, the duration of which varied randomly on any given trial from 1 to $40 \mathrm{sec}$. The animal was required to nosepoke (NP) in the photocell device on the opposite wall at least once during the Delay interval signaled by the presence of the illuminated cue light. The last nosepoke (LNP) that occurred after the delay interval had timed out on a given trial turned off the cue light and extended both levers into the chamber, designating the onset of the Nonmatch or Recognition phase of the task. At this time the animal was required to press the lever opposite to the SR [i.e., a Nonmatch response (NR)] for a reward, which was signaled immediately by a distinct "click" of the water delivery solenoid and appearance of water in the trough next to the lever. The levers then were retracted for the $10 \mathrm{sec}$ intertrial interval (ITI). On correct trials, water was consumed (Reinforcement phase) during this $10 \mathrm{sec}$ period. On incorrect (error) trials, an inappropriate (i.e., "match") lever press was followed by an immediate $5 \mathrm{sec}$ time-out period in which the levers retracted, and the house lights were turned off, leaving the chamber completely dark. After this time-out period, the lights were turned back on for an additional $5 \mathrm{sec}$. A new trial was initiated by the extension of one of the levers selected at random, a total of $10 \mathrm{sec}$ (ITI) after the Recognition phase response (correct or error).

The average time required to train a naive animal to criterion in the DNMS task with $1-40 \mathrm{sec}$ delays was $\sim 1$ month. Training involved several phases in which different procedures were used to develop selective responding on each lever, stimulus control over nosepoke responding during the delay, and linkage of responding in the Sample and Recognition phases of the task. Once animals were able to respond to all facets of a single DNMS trial appropriately, with no delay, they were moved out rapidly with respect to exposure to trials with increasing durations of the delay, first at delays of 1-15 sec and then 1-30 sec or 1-40 sec delay trials. A final criterion of $>85 \%$ correct responding on trials with delays of 1-5 sec during sessions with 1-30 or 1-40 sec delay trials was used for all animals (Hampson et al., 1993; Deadwyler et al., 1996).

Drug preparation and administration. CX516 was prepared as a 35 $\mathrm{mg} / \mathrm{ml}$ stock in a cyclodextrin vehicle. The vehicle solution was mixed as $25 \% \mathrm{wt} / \mathrm{vol}$ solution by adding $2.5 \mathrm{gm}$ 2-hydroxypropyl- $\beta$-cyclodextrin (Research Biochemicals, Natick, MA) to $5.0 \mathrm{ml}$ sterile saline $(0.9 \%)$ and diluted to $10.0 \mathrm{ml}$ total volume with sterile deionized water. Drug solutions were prepared by adding $35 \mathrm{mg}$ of CX516 (Cortex Pharmaceuticals, Irvine, CA) powder to $1.0 \mathrm{ml}$ of cyclodextrin vehicle. Both drug and vehicle solutions were sonicated at high power for $15 \mathrm{sec}$ to ensure thorough dissolution and mixing of the vehicle and drug. On drug administration days, animals were injected intraperitoneally with the CX516/cyclodextrin solution $(1 \mathrm{ml} / \mathrm{kg}$ of the $35 \mathrm{mg} / \mathrm{ml}$ solution to provide $35 \mathrm{mg} / \mathrm{kg}$ ) $\sim 5 \mathrm{~min}$ before the start of the behavioral session. On vehicle-only days, the vehicle solution was administered at $1 \mathrm{ml} / \mathrm{kg} 5 \mathrm{~min}$ before the start of the session. All CX516 solutions were mixed fresh each day. Vehicle solutions were mixed and maintained over $5 \mathrm{~d}$, stored at $4^{\circ} \mathrm{C}$, and warmed to room temperature and sonicated to remix the cyclodextrin before daily use.

Behavioral analyses. Analysis of behavioral data consisted of several different measures designed to elucidate different DNMS performance factors. The two primary measures used to test performance differences were mean percent correct trials during the session and mean percent correct trials at each delay interval assessed in $5.0 \mathrm{sec}$ blocks. Additional measures included time of execution of the trial and influence of previous trial delay (Hampson and Deadwyler, 1996b; Deadwyler and Hampson, 1997). ANOVA was used for most comparisons, with adjusted pairwise contrasts used for individual comparisons and simple effects. Trial-to- 


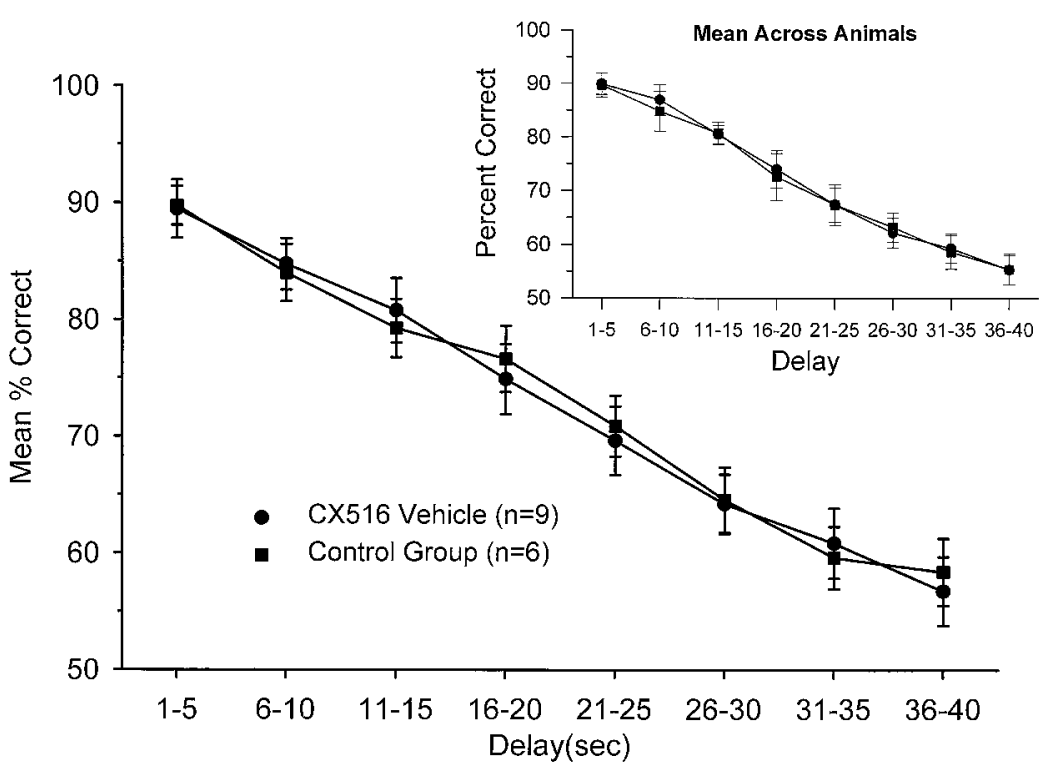

Figure 1. Behavioral performance in the DNMS task for CX516 $(n=9)$ and Control $(n=6)$ groups on days $1-8$, before drug administration. Each group was injected with cyclodextrin vehicle for $8 \mathrm{~d}$ before the $17 \mathrm{~d}$ drug treatment. Mean (and SEM) percent correct performance was calculated over all trials and animals within $5 \mathrm{sec}$ delay increments, and plotted for both the CX516 and Control groups. The near-linear slope indicates the dependence of DNMS performance on the length of the delay interval in both groups. The inset shows the same DNMS performance data in which means for each were calculated and then averaged across animals, reflecting the absence of interanimal differences.

trial influences were examined by various methods of sorting the data as a function of performance on the previous trial or in terms of delay intervals on any given trial.

\section{RESULTS}

\section{Baseline DNMS performance before administration of CX516}

All animals $(n=18)$ were trained to criterion DNMS performance at delays of either $1-30(n=7)$ or $1-40 \mathrm{sec}(n=11)$. As noted above, the study was conducted on three separate groups of animals tested at three different intervals over a 2 year period. In each case the exact same training and testing protocols were used (with the exception of maximum length of trial delay). All animals were injected with vehicle $5 \mathrm{~min}$ before the start of the session and trained at criterion for 8-12 d before drug treatment. Performance during this period was stable at a mean of $72.7 \pm$ $2.7 \%$ (mean \pm SEM) for the control group and $72.9 \pm 2.6 \%$ for the CX516 group across all delays. No differences with respect to either replication (subgroups tested at different times) or vehicle were detected. Performance at each delay was grouped into $5 \mathrm{sec}$ intervals from 1 to $40 \mathrm{sec}$ and assessed as a function of the duration of the delay period. Mean performance across the various delay intervals is shown in Figure 1 for the last $8 \mathrm{~d}$ of criterion performance for each group. Because interanimal differences could have been obscured by pooling trials across animals, the inset to Figure 1 shows the overall mean and SEMs calculated differently, across the average performance of each animal at each delay. There were no significant differences in DNMS performance as a function of delay $\left(F_{(17,742)}=0.32\right.$; NS) between the two groups by either measure during this pretreatment period. However, there was a marked decrease from 90 to $58 \%$ correct responses as a function of (1-40 sec) delay interval $\left(F_{(7,742)}=6.17 ; p<0.001\right)$ assessed across both groups. Chance (random) performance was $50 \%$ correct responses, and at delays of $>35 \mathrm{sec}$ (Fig. 1) performance was near random levels, demonstrating the critical influence of length of Delay (Hampson and Deadwyler, 1996b).

\section{Effects of CX516 on DNMS performance}

CX516 was administered over a 17 d treatment regimen, alternating with days in which only vehicle and no drug was given.
Control group animals received injections of vehicle every day and were tested over the same time course. Because three different "squads" of animals were run repeatedly in this protocol at different times, control animals (vehicle only) were always "paired" with animals given drug in each replication of the experiment. Each CX516-treated animal had the same number of Pre-CX516 vehicle (days 1-8), CX516 (days 9-24), and PostCX516 vehicle (days 25-32) sessions. Animals in the Control group provided an assessment of changes in baseline DNMS performance over the $32 \mathrm{~d}$ testing period as well as a comparison with the CX516 group. There were no significant differences in performance between the three sets of independently tested animals (Fig. 1, inset); therefore the data were pooled into a single Control group $(n=6)$ and a single CX516-treated group $(n=12)$.

Facilitation of DNMS performance by CX516 was manifested as a significant increase in the mean number of correct responses on trials when calculated across all delay intervals $(1-40 \mathrm{sec})$ in comparison with both the Control group over the same testing period $\left(F_{(7,742)}=2.71 ; p<0.01\right)$ and Pre-CX516 performance $\left(F_{(7,742)}=3.46 ; p<0.01\right)$. Figure 2 shows the effects of CX516 on drug days in comparison with the Control group averaged over the $17 \mathrm{~d}$ alternating drug treatment period. The open symbols denote performance on days in which CX516 was administered; filled circles indicate performance of the same group on alternate vehicle-only days. Asterisks indicate delays that were significantly increased over Control animals by linear contrasts within the ANOVA (**, $\left.F_{(1,742)}>11.03, p<0.001\right)$. Performance was significantly $(p<0.001)$ enhanced by CX516 versus Control animals during this period except for the shortest (1-5 sec) and longest (36-40 sec) delays.

\section{Time course of CX516 facilitation of DNMS performance}

DNMS performance was significantly elevated within the first $3 \mathrm{~d}$ of exposure to CX516 compared with the Pre-CX516 baseline (days 1-8). Baseline performance (72\%) was calculated over days 1-8 for both groups (see Materials and Methods). Changes in performance were plotted as percent maximum increase from this control level, with the baseline at $0 \%$ (no increase) and a 


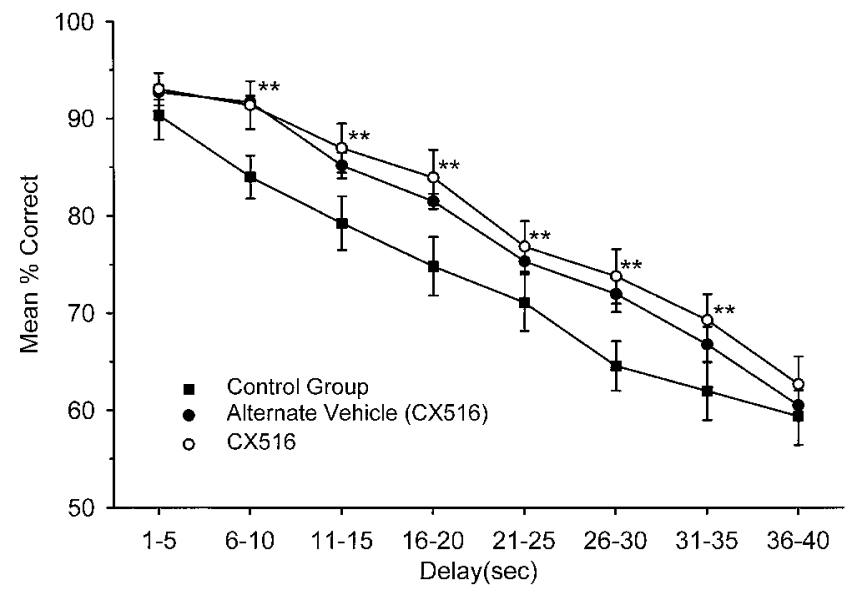

Figure 2. Mean percent correct performance as a function of delay for CX516 and Control groups averaged over days 9-25. Curves were calculated for the nine sessions during which drug $(35 \mathrm{mg} / \mathrm{kg})$ was administered (open circles) to the CX516 group, and separately for the 8 alternate vehicle days for the same group (filled circles). Curve with filled squares shows performance over the same $17 \mathrm{~d}$ period for the six animals in the Control group that received only vehicle injections. Trials were sorted by delay as in Figure 1 and plotted as mean $( \pm$ SEM) performance across all trials and animals. Asterisks indicate significantly $\left({ }^{* *} p<0.001\right)$ different performance from Control group.

maximum increase of $25 \%$ over baseline (corresponding to $\sim 88 \%$ Correct DNMS responses). Figure 3 shows this change over the entire course of testing for CX516-treated versus Control animals. As in Figure 1 there were no significant differences in mean baseline performance between the CX516 and Control group means on days $1-8\left(F_{(1,497)}=0.76\right.$; NS $)$. However, by the second CX516 day (day 11 of the overall regimen) (Fig. 3, asterisk), performance of CX516-treated animals was significantly above that of the Control group $\left(F_{(1,497)}=7.41 ; p<0.01\right)$ and remained so for the rest of the study. Control group performance increased $\leq 5 \%$ over the entire $32 \mathrm{~d}$ of testing, whereas the CX516-treated animals showed a marked and progressive increase in performance (to a maximum of $25 \%$ from Pre-CX516 performance) over the $17 \mathrm{~d}$ drug administration period (Fig. 3, vertical lines). The mean ( \pm SEM) increase for the CX516 group was $14.3 \pm 3.5 \%$ over days $9-25$, compared with only $1.9 \pm 1.5 \%$ for the Control group over the same time interval $\left(F_{(9,497)}=3.41\right.$; $p<0.001)$.

Figure 3 shows that CX516-treated animals exhibited increased levels of performance to a relative asymptote at the maximum increase of $88 \%$ correct after CX516. Drug treatment was suspended on day 25 to determine whether improvement would decrease or persist at the same elevated level. Days 26-32 in Figure 3 show that the CX516-treated group maintained the same level of performance achieved during drug treatment (day 25) throughout the $7 \mathrm{~d}$ after drug testing, which was markedly different from the Control group (mean difference $=15.3 \pm 0.7 \%$; $\left.F_{(1,497)}=21.65 ; p<0.001\right)$.

\section{Carryover of CX516 effects to nondrug testing days}

A most unusual effect of CX516 administration was that performance also remained elevated on the intervening vehicle-only days when no drug was injected. This "carryover" effect is shown in Figure 2 (unfilled circles) as a function of delay interval, and in Figure 3 where performance in the CX516-treated group remained elevated on the intervening vehicle days (filled circles) at

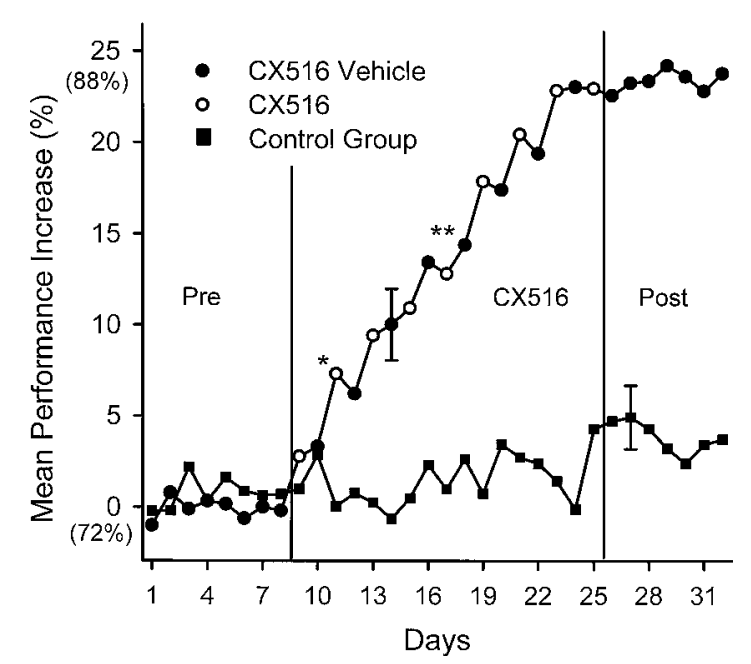

Figure 3. Mean percent change in overall DNMS performance is shown for the CX516 (circles) and Control (squares) group over the entire $32 \mathrm{~d}$ treatment period. Mean DNMS performance over all trials was calculated for each individual session and transformed to percent increase from baseline performance. Mean baseline performance was $72 \%$ over days $1-8$. The maximum level corresponded to a mean of $88 \%$ correct (days 26-32). Each point is the mean performance over all animals within each group for that day. The error bar indicates the largest SEM across all days. Pre, CX516, and Post indicate drug administration $(35 \mathrm{mg} / \mathrm{kg})$ to CX516 on alternate days (open circles). On all other days (closed circles), animals received vehicle only. Control group (filled squares) received vehicle on all $32 \mathrm{~d}$. Asterisks indicate significant increases from Control group on that day $\left({ }^{*} p<0.01 ;{ }^{* *} p<0.001\right)$.

the same level as previous drug injection days (open circles). Statistical comparisons showed no significant differences $\left(F_{(1,497)}\right.$ $=1.09$; NS) between the two. Thus the effects of CX516 were cumulative over days and persisted on intervening vehicle-only days when the drug was not administered.

\section{Differential effect of CX516 on long versus short delay trials}

Figure 4 shows mean daily performance plotted for the CX516 group with respect to trials with different delay intervals. These are the same data shown in Figure 3, only they are grouped into trials with various delay lengths ( 5 or $10 \mathrm{sec}$ intervals) and plotted separately for each day of testing. For clarity, the 21-25 and 26-30 sec intervals delays are combined into a single 21-30 sec curve, and 36-40 sec delays (which showed no significant change) (Fig. 2) were not included in Figure 3. Performance increases shown in Figure 4 are relative to baseline performance within each delay group calculated over days 1-8 (mean percent correct DNMS trials: $1-5 \mathrm{sec}=92 \%, 6-10 \mathrm{sec}=86 \%, 11-15 \mathrm{sec}=84 \%$, $16-20 \mathrm{sec}=76 \%, 21-30 \mathrm{sec}=68 \%, 31-35 \mathrm{sec}=62 \%)$. It is quite clear that no significant improvement occurred on the 1-5 sec delay trials as shown in Figure 2. Clearly, performance was enhanced in the CX516 group on all trials with $>5 \mathrm{sec}$ delays. The maximum percent change across days $8-25$, in comparison with predrug vehicle levels, was on trials with 31-35 sec delays $\left(54.5 \% ; F_{(1,497)}=25.17 ; p<0.001\right)$, whereas the least significant increase $\left(10 \% ; F_{(1,497)}=7.39 ; p<0.01\right)$ (Fig. 4, asterisk) occurred on trials with $6-10 \mathrm{sec}$ delays. This differential improvement as a function of delay also persisted in the CX516 group after termination of the drug on days 26-32 (Fig. 4). The Control group showed no significant increases in performance as a function of delay interval on any of the above comparisons over the same 


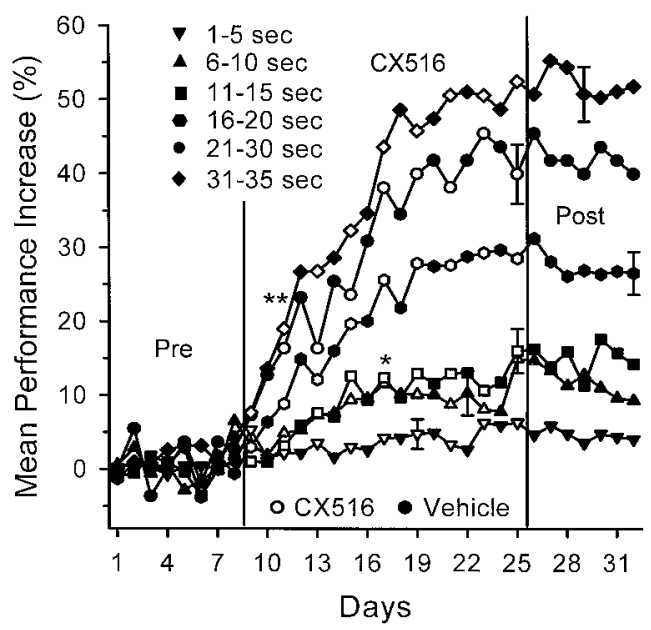

Figure 4. Mean percent change in DNMS performance over days for animals receiving CX516 $(n=9)$ plotted for trials sorted by length of the delay interval (in $5 \mathrm{sec}$ intervals). Trials with $21-25$ and $26-30 \mathrm{sec}$ delays were combined as $21-30 \mathrm{sec}$ delays, because the two intervals did not differ in mean performance increase. The 36-40 sec delays are not shown because they were similar to the $1-5 \mathrm{sec}$ data, which were not significantly increased over control levels (Fig. 2). Performance was plotted as percent increase from baseline before drug administration (see Fig. 3), with the baseline $(0 \%)$ calculated separately for each delay (see Results). Error bars indicate the largest SEM within each curve. Open symbols indicate days on which CX516 was administered; filled symbols indicate vehicle administration. Asterisks indicate significant increase from Control group on that day $\left({ }^{*} p<0.01 ;{ }^{* *} p<0.001\right)$. Pre, CX516, and Post are as indicated in Figure 3.

testing period $\left(F_{(31,497)}=0.21 ; \mathrm{NS}\right)$. The latter finding indicates that drug-related improved performance was not the result of increased training on the task. Thus, the major contribution to the overall performance increase by the CX516 group depicted in Figure 2 was a differential as well as selective improvement on trials within the range of $6-35 \mathrm{sec}$.

Figure 5 shows performance by delay curves for the Control and CX516 groups summed over days 26-32 in which only vehicle was administered. The same data are also plotted as the mean of each individual animal's performance at each delay (inset) to demonstrate that interanimal variability was not a factor in these effects. It is clear that the animals receiving CX516 $(n=9)$ exhibited superior performance $\left(F_{(2,742)}=8.71 ; p<0.001\right)$ across all delays (except the $1-5 \mathrm{sec}$ interval) in comparison with the Control group $(n=6)$.

\section{CX516 selectively eliminated error factors in the spatial DNMS task}

The above finding suggests that CX516 had a differential influence on performance at long versus short delay trials. Previous error analyses of performance in this task revealed two main types of errors: (1) those associated with incorrect encoding or "miscoding" of Sample position that are independent of delay, and (2) delay-dependent errors that result from "weak" encoding of the Sample, provoking susceptibility to loss of information across long delay intervals (Deadwyler et al., 1996). There is, in fact, a strong interaction between the two error types. The occurrence of errors on long delay trials [long delay errors (LDEs)] has been shown to proactively interfere with performance on the subsequent trial in this version of the DNMS task (Hampson and Deadwyler, 1996b).

A detailed analysis of this effect showed that interference

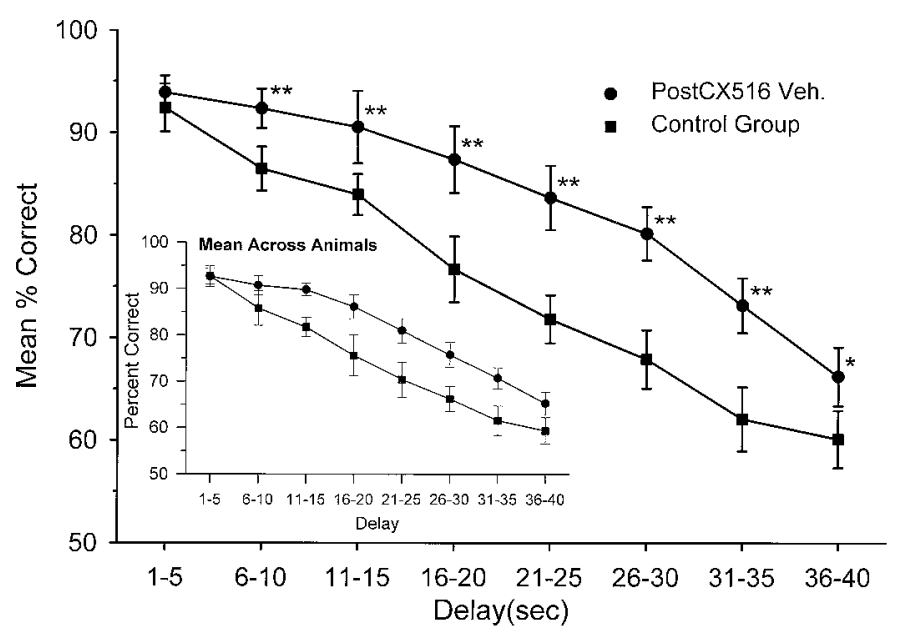

Figure 5. Mean performance curves for CX516 and Control group over days 26-32 (PostCX516 Veh.) indicate that behavioral performance remained elevated for at least 1 week after drug was discontinued. Mean DNMS performance (see Fig. 3) for all trials across all animals at a given delay for the CX516-treated (circles) and vehicle-only Control (squares) groups. The inset indicates the mean (and SEM) across animals for the same data (see Fig. 3). Asterisks indicate significant performance increases within the $5 \mathrm{sec}$ intervals compared with Control group $\left({ }^{*} p<0.01\right.$; ${ }^{*} p<$ 0.001).

increased as a function of length of delay in the preceding trial, primarily because LDEs exhibited higher frequency at delays $>15$ sec. The analysis of both behavioral and hippocampal neuron responses demonstrated that animals have a strong tendency to repeat the erroneous Nonmatch response (NR) if the previous trial was an LDE. Thus the SR made on the next trial is "miscoded" with the position of the incorrect NR in the Recognition phase on the previous LDE trial when the position of the Sample lever presented is not the same as the LDE. This shift to a maximizing strategy forces a deliberate error (miscode) $50 \%$ of the time, because only half of the trials presented will be compatible with the "biased" code for the SR from the previous LDE (Deadwyler et al., 1996). Extensive analyses (Hampson and Deadwyler, 1996b) have shown that the shift to the maximization strategy after an LDE actually increases the probability that the animal will be correct more often than if it attempted to do the trial in the normal (DNMS) manner. We have speculated that this strategy shift provides a means for the animal to avoid "strings" or "runs" of LDE trials. The latter assumption follows from the fact that miscoded Samples are encoded strongly, apparently to "survive" trials of any delay (Hampson and Deadwyler, 1996a,b).

Implementation of the maximizing strategy on trials preceded by LDEs gave rise to the symmetric appearance of the same/ different performance curves shown in Figure 6 (circles), in which performance was almost completely dependent on the position of error made in the previous trial $(p<0.001)$. If the trial exceeded $15 \mathrm{sec}$ (i.e., an LDE), the two curves (same and different) were a strict function of the probability that the trial after an LDE matched the position of the NR on the previous LDE trial. If the preceding error trial was $<15 \mathrm{sec}$, performance was nearly random, but still slightly influenced by the preceding NR response (Fig. 6). Because there were only two types of Sample-Nonmatch trial in the task (L-R or R-L, respectively), the performance curve is symmetric around the $50 \%$ (chance) level. We have shown previously that the animal "miscodes" $50 \%$ of trials after LDEs, regardless of which Sample lever is actually presented, 


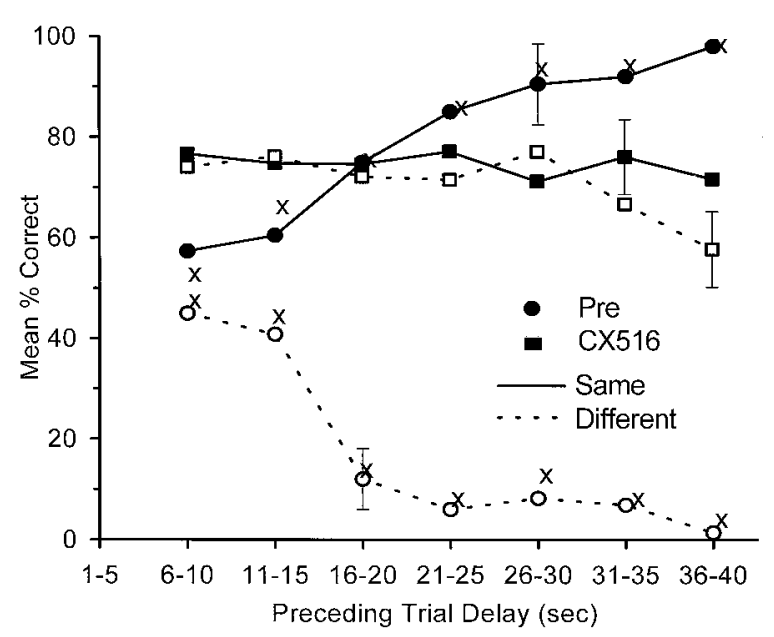

Figure 6. DNMS performance before and after CX516 treatment only on trials after error trials plotted as a function of length of delay on the preceding error trial. Open and filled symbols indicate mean (and SEM) performance from the nine animals in the CX516 treatment group. Performance on trials was also sorted according to whether the preceding error trial was the same (filled symbols, solid lines) or different (open symbols, dashed lines) with respect to Sample, as the current trial on which performance is plotted. Only trials that followed an error trial are plotted (errors did not occur at 1-5 sec delays). Circles: Pre-CX516 sessions from days $1-8$ for the nine animals in the CX516 group. The equivalent performance for the six animals in the Control group across all days is indicated by $X$ points. Squares: Post-CX516 sessions from days 26-32. The symmetric separation of the Same and Different performance curves indicates total dependence on the type of trial presented relative to the previous error trial. After CX516 treatment, performance dependence on previous error trial is eliminated.

because there is only one chance in two that the lever presented will match the previous NR error.

In marked contrast to Pre-CX516 and Control group performance, no proactive interference occurred in animals assessed during or after CX516 treatment. Figure 6 (squares) shows that the same/different performance curves (over days 26-32) were not statistically different $\left(F_{(10,554)}=1.07\right.$; NS) after an LDE of any duration and that the significant increase in overall performance on both trial types was independent of preceding delay $($ mean Pre-CX516 $=50.35 \pm 37.5 \%$; mean CX516 $=74.4 \pm$ $\left.11.9 \% ; F_{(3,554)}=5.76 ; p<0.001\right)$. Thus, proactive interference and the maximization strategy shift were eliminated in animals treated with CX516.

\section{Partial effects of CX516 on DNMS performance}

Figures 3 and 4 clearly demonstrate that the effects of CX516 persisted when animals were tested on subsequent intervening vehicle days. However, this "carryover" influence of CX516 to the next (nondrug) intervening vehicle session was not observed in all animals injected with CX516. Three of 12 animals given CX516 showed elevations nearly equivalent to those of the other nine animals but no "carryover" to the intervening vehicle sessions (Fig. 7). Close examination revealed that significant elevations in performance in these three animals did not persist beyond the first half (30-45 $\mathrm{min})$ of the drug day testing session. There were in fact no significant differences on drug days between the first half session mean performance of these three "noncarryover" animals and the means over the whole session of the "carryover" group $\left(F_{(17,497)}=0.79 ; \mathrm{NS}\right)$, as shown in Figure 7 , whereas performance in the second half of each drug session was significantly reduced for the same three animals $\left(F_{(1,277)}=9.64 ; p<\right.$

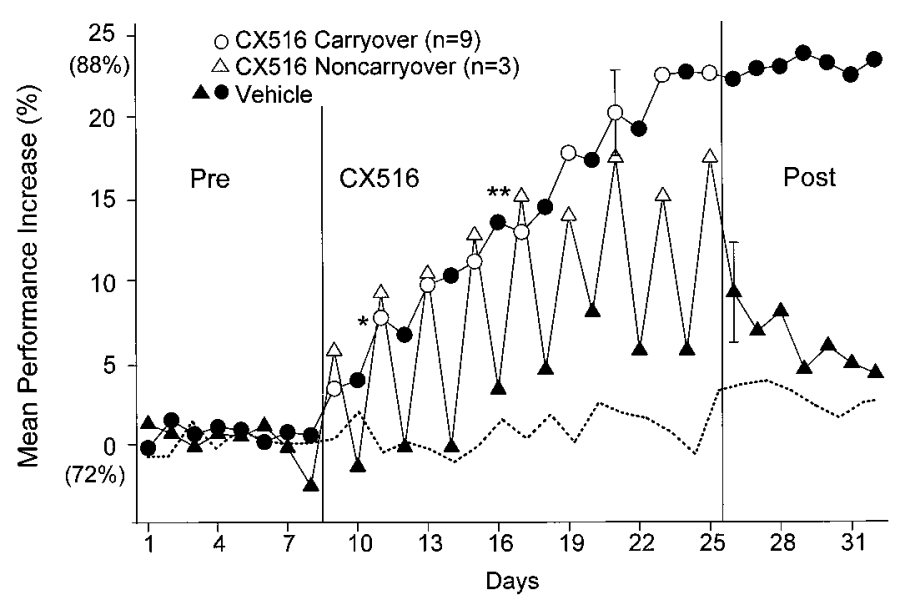

Figure 7. Mean percent increase in overall DNMS performance is shown for noncarryover animals. The open triangles show performance calculated only across the first 50 trials (i.e., first half) of each CX516 sessions. Filled triangles show performance on interposed vehicle sessions. Circles (open and closed) replot DNMS performance of carryover group from Figure 3. Dashed line indicates performance over the same period for Control group given vehicle-only injections. DNMS performance is plotted as mean percent increase in performance from baseline (PreCX516) (see Fig. 3). There was no differentiation of DNMS performance for the first versus second half of noncarryover vehicle session. Error bars indicate the largest SEM within each group. Vertical lines demarcate CX516 treatment days. Pre, CX516, and Post are as indicated in Figure 3. Asterisks indicate performance levels significantly increased from Control group on that day $(* p<0.01 ; * * p<0.001)$.

0.01). Performance in the first half of the session increased progressively across days $9-18$ to the same degree as that of the other nine carryover animals (mean performance increase: carryover, $11.9 \pm 1.8 \%$, noncarryover, $13.1 \pm 1.9 \% ; F_{(1,497)}=0.38$; NS) until days 19-25, when performance in the carryover group increased above that of the noncarryover animals (carryover mean increase $=25.7 \pm 1.4 \%$, noncarryover $=19.6 \pm 1.1 \%$; $\left.F_{(1,497)}=4.02 ; p<0.05\right)$.

Figure 7 shows that the three noncarryover animals exhibited significantly elevated performance (mean increase $=11.4 \%$; $\left.F_{(1,497)}=6.92 ; p<0.01\right)$ on intervening vehicle days relative to the Control group over the same days of testing (days 8-17) (Figs. $3,4)$. Comparison of the second half of the CX516 treatment session for these three animals with the intervening vehicle days was not significantly different $\left(F_{(1,277)}=2.01\right.$; NS). Finally, in contrast to the carryover group, the three noncarryover animals showed a significant $\left(F_{(1,497)}=6.99 ; p<0.01\right)$ decline in mean performance over days 26-32 when CX516 administration was terminated (Fig. 7). This change in performance level between the first and second half of the session (35-40 $\mathrm{min})$ in the noncarryover animals is supported by the time course of CX516 actions in behaving animals, determined by its pharmacokinetic profile (Staubli et al., 1994a; Rogers, 1997).

\section{DISCUSSION}

These results on the effects of the ampakine CX516 (also known as BDP-12) on DNMS performance share a high degree of consistency with previous reports of the facilitative effects of this and similar compounds on other behavioral tests (Shors et al., 1994; Staubli et al., 1994b; Larson et al., 1995; Granger et al., 1996). In this regard, the use of CX516 has served as both an independent variable to be investigated relative to its enhancing effect on DNMS behavior and as a pharmacological "tool" to gain 
further insight into the behavioral and cognitive processes operative during successful DNMS performance. Previous studies have shown that ampakines improve retention in tasks involving delays of hours to days (Staubli et al., 1994b; Larson and Vanderklish, 1997). The present findings confirm and extend those findings to a task with well defined delay intervals and more intense "short-term memory" requirements (Hampson et al., 1993; Deadwyler et al., 1996).

\section{Dose-effect of CX516 on behavioral performance}

The $35 \mathrm{mg} / \mathrm{kg}$ dosage of CX516 used in the present experiments is similar to that used previously (range, $30-50 \mathrm{mg} / \mathrm{kg}$ ) and results in blood levels $(50 \mu \mathrm{M})$ that closely match brain levels (Rogers, 1997). The equivalent concentration of CX516 is sufficient to increase the efficacy of monosynaptic potentials in brain slices (Staubli et al., 1994b) and is well above threshold (10-15 $\mu \mathrm{M})$ for producing reliable changes in polysynaptic glutamatergic responses in vivo (Arai and Lynch, 1992). The dose used here (35 $\mathrm{mg} / \mathrm{kg}$ ) improves recall in rats after 6 or $8 \mathrm{hr}$ delays in a radial arm maze (Staubli et al., 1994b) and reduces the number of trials needed to form stable two-odor discriminations in rats (Larson et al., 1995). Dose-effect studies in this DNMS task revealed that higher doses $(50-70 \mathrm{mg} / \mathrm{kg})$ produced a facilitation of performance, but the animals periodically failed to complete the 100200 trial session. Doses below $35 \mathrm{mg} / \mathrm{kg}(10-20 \mathrm{mg} / \mathrm{kg})$ were also effective in facilitating DNMS performance, but they were more inconsistent across animals than the $35 \mathrm{mg} / \mathrm{kg}$ dose.

\section{Enhancement of DNMS performance by repeated exposure to CX516}

Performance of the DNMS task improved on a day-to-day basis during exposure to the ampakine CX516, over the $17 \mathrm{~d}$ drug/ vehicle treatment period. A pronounced improvement in delaydependent performance (Figs. 2, 5), as well as a differential degree of facilitation on trials with long versus short delay intervals (Figs. 4, 5), were key factors relating to the effects of CX516. Overall performance in the carryover group was improved by almost $25 \%$ relative to Pre-CX516 levels, either in the same animals or when compared with vehicle Control animals. The effects of CX516 on performance were incremental, showing on average a $4 \%$ improvement per daily (drug) session, and persisted for 7 additional days of testing after termination of drug treatment (Figs. 3, 5). A subgroup of three animals (noncarryover) treated identically with CX516 showed drug-related improvement in DNMS performance on drug days, but task facilitation was brief and lasted only through the first half (35-40 $\mathrm{min}$ ) of the session (Fig. 7). Comparison of carryover and noncarryover groups revealed that incremental improvement on drug days (at least in the first $5 \mathrm{~d}$ of CX516 exposure) clearly did not depend on sustaining drug-related performance levels on the intervening vehicle days. Noncarryover animals showed markedly reduced residual effects of the drug on intervening vehicle days, as well as lower asymptotic performance levels. This suggests that the ampakine, when present, exerted an influence that could be differentiated from days in which the drug was not present.

\section{Carryover effects of CX516 on DNMS performance}

Previous studies have shown that CX516 is metabolized relatively rapidly by rats, with a half-life in blood of $\sim 15-20 \mathrm{~min}$ and a range of about twice that (Rogers, 1997). A major metabolite of CX516, which exhibits one third the behavioral potency, has been identified and synthesized and has nearly the same half-life as the parent drug. Therefore, a dose of $35.0 \mathrm{mg} / \mathrm{kg}$ in an animal of average weight would produce significant levels of active drug or metabolites in plasma and brain for $\sim 2 \mathrm{hr}$, with a steady decline from $\sim 40 \mathrm{~min}$ after injection (Rogers, 1997). There is no evidence at this time of either irreversible or long-term binding of CX516 in rat brain. Thus, unlike the three animals shown in Figure 7, the carryover effect of CX516 is not predictable from its pharmacokinetic profile and hence not likely caused by the continued presence of the drug or a long-lived metabolite.

It is possible that the carryover effect of the ampakine is attributable to the use of overtrained animals and/or a protracted period of drug treatment, features that are prominent aspects of the present study and absent in many previous reports. However, experiments involving radial mazes (Granger et al., 1993, 1996; Staubli et al., 1994a,b) and simultaneous odor discriminations (Staubli et al., 1994a; Larson et al., 1995) did use well trained rats and repeated injections but still apparently found no evidence of cumulative effects with CX516 at the dosage used here. One possible explanation is that the ampakine enhanced the learning of new and more efficient means of dealing with the DNMS contingency. All animals were at asymptotic levels of performance before initiation of CX516 treatments. The Control group, run in parallel, did not show significant improvements over the drug testing period. Therefore it is plausible to assume that the animals receiving CX516 day by day learned new aspects of the DNMS task not possible in the absence of drug. The cumulative nature of the drug effect, i.e., small incremental gains, is actually a feature more reminiscent of procedural learning (Squire, 1992) and as such would account for the persistence of the improvement in the absence of the drug. Thus, biochemical, anatomic, and molecular changes set in motion by the drug could well have initiated new or additional learning (that does not occur normally) in the task. Evidence that the latter condition in fact was responsible for improved performance was revealed by the CX516 elimination of proactive interference.

\section{Elimination of proactive interference by CX516}

In rather detailed previous analyses, we have characterized trialspecific factors responsible for two major types of errors in this version of the spatial DNMS task (Deadwyler et al., 1996; Deadwyler and Hampson, 1997). One major factor that accounts for $30 \%$ of total errors committed by animals engaged in the task is proactive interference from one trial to the next (Hampson and Deadwyler, 1996b).

The hippocampus plays a major role in suppressing proactive interference in this type of task, as shown by studies using selective and reversible hippocampal lesions (Dunnett, 1985; Heyser et al., 1993; Hampson et al., 1995). Animals with hippocampal lesions are susceptible to proactive interference on all trials regardless of the delay, whereas intact animals are susceptible only if the preceding trial was a long delay error (LDE). Under nondrug conditions, an intact hippocampus can protect against proactive influences, but only if preceding trials do not exceed $15 \mathrm{sec}$ and they are not errors (i.e., they are not LDEs); hence Figure 5 shows that a substantial number of errors occur on trials with delays $>15$ sec. The application of the maximizing strategy and the subsequent miscode error therefore could occur on a trial with any delay. The miscode is made in the Sample phase of the trial immediately after an LDE trial (Hampson and Deadwyler, 1996a,b; Hampson et al., 1998). Figure 6 also shows that CX516-treated animals became much less susceptible to the interfering effects of previous trials, whereas Control animals continued to be affected. Suppression of this proactive influence 
by the ampakine did not result from elimination of LDE trials, because they continued to occur (albeit with a reduced frequency). Rather, after exposure to CX516, the maximizing strategy that normally occurred on trials after LDE trials did not occur.

The behavioral effects of CX516 described above were accompanied by changes in simultaneously recorded hippocampal cell activity in the same animals. In the companion article (Hampson et al., 1998), several of the above behavioral aspects of the facilitative effects of CX516 are explored in detail through presentation and description of hippocampal cell firing correlates recorded from these same animals throughout the course of CX516 testing.

\section{REFERENCES}

Arai A, Lynch G (1992) Factors regulating the magnitude of long-term potentiation induced by theta pattern stimulation. Brain Res 598:173-184.

Arai A, Silberg J, Kessler M, Lynch G (1995) Effect of thiocyanate on AMPA receptor mediated responses in excised patches and hippocampal slices. Neuroscience 66:815-827.

Deadwyler SA, Hampson RE (1997) The significance of neural ensemble codes during behavior and cognition. In: Annual review of neuroscience (Cowan WM, Shooter EM, Stevens CF, Thompson RF, eds), pp 217-244. Palo Alto, CA: Annual Reviews.

Deadwyler SA, Bunn T, Hampson RE (1996) Hippocampal ensemble activity during spatial delayed-nonmatch-to-sample performance in rats. J Neurosci 16:354-372.

Dunnett SB (1985) Comparative effects of cholinergic drugs and lesions of nucleus basalis or fimbria-fornix on delayed matching in rats. Psychopharmacology 87:357-363.

Dunnett SB (1989) Comparison of short-term memory deficits in animal models of aging using an operant delayed response task in rats. In: The biology of memory (Squire LR, Lindenlaub E, eds), pp 581-603. New York: FK Schattauer Verlag.

Eichenbaum H, Kuperstein M, Fagan A, Nagode J (1987) Cue-sampling and goal-approach correlates of hippocampal unit activity in rats performing an odor discrimination task. J Neurosci 7:716-732.

Granger R, Staubli U, Davis M, Perez Y, Nilsson L, Rogers GA, Lynch G (1993) A drug that facilitates glutamatergic transmission reduces exploratory activity and improves performance in a learning-dependent task. Synapse 15:326-329.

Granger R, Deadwyler SA, Davis M, Moskovitz B, Kessler M, Rogers G, Lynch G (1996) Facilitation of glutamate receptors reverses an ageassociated memory impairment in rats. Synapse 22:332-337.

Hampson RE, Byrd DR, Konstantopoulos JK, Bunn T, Jarrard LE, Deadwyler SA (1995) Proactive interference and short-term memory during performance of a DNMS task in normal rats and rats with hippocampus removed. Soc Neurosci Abstr 21:1215.

Hampson RE, Deadwyler SA 1996a LTP and LTD and the encoding of memory in small ensembles of hippocampal neurons. In: Long-term potentiation, Vol 3, Ed 3 (Baudry M, Davis J, eds), pp 199-214. Cambridge, MA: MIT.

Hampson RE, Deadwyler SA 1996b Ensemble codes involving hippocampal neurons are at risk during delayed performance tests. Proc Natl Acad Sci USA 93:13487-13493.

Hampson RE, Heyser CJ, Deadwyler SA (1993) Hippocampal cell firing correlates of delayed-match-to-sample performance in the rat. Behav Neurosci 107:715-739.

Hampson RE, Rogers G, Lynch G, Deadwyler SA (1998) Facilitative effects of the ampakine CX516 on short-term memory in rats: correlations with hippocampal neuronal activity. J Neurosci 18:2748-2763.

Heyser CJ, Hampson RE, Deadwyler SA (1993) The effects of delta-9THC on delayed match to sample performance in rats: alterations in short-term memory produced by changes in task specific firing of hippocampal neurons. J Pharmacol Exp Ther 264:294-307.

Isaacson JS, Nicoll RA (1991) Aniracetam reduces glutamate receptor desensitization and slows the decay of fast excitatory synaptic currents in the hippocampus. Proc Natl Acad Sci USA 88:10936-10940.

Ito I, Tanabe S, Kohda A, Sugiyama H (1990) Allosteric potentiation of quisqualate receptors by a nootropic drug aniracetam. J Physiol (Lond) 424:533-543.

Larson J, Lynch G (1988) Role of $N$-methyl-D-aspartate receptors in the induction of synaptic potentiation by burst stimulation patterned after the hippocampal theta-rhythm. Brain Res 441:111-118.

Larson J, Vanderklish PW (1997) Involvement of AMPA receptors in LTP mechanisms and memory. In: Long-term potentiation, Vol 3 (Baudry M, Davis JL, eds), pp 73-104. Cambridge, MA: MIT.

Larson J, Lieu T, Petchpradub V, LeDuc B, Ngo H, Rogers GA, Lynch G (1995) Facilitation of olfactory learning by a modulator of AMPA receptors. J Neurosci 15:8023-8030.

Lynch G, Kessler M, Rogers G, Ambros-Ingerson J, Granger R, Schehr RS (1996) Psychological effects of a drug that facilitates brain AMPA receptors. Int Clin Psychopharmacol 11:12-19.

Morris RG, Anderson E, Lynch GS, Baudry M (1986) Selective impairment of learning and blockade of long-term potentiation by an $N$-methyl-D-aspartate receptor antagonist, AP5. Nature 319:774-776.

Rogers GA (1997) XIII International Symposium on Radiopharmaceutical Chemistry, Uppsala, June.

Shors TJ, Servatius RJ, Thompson RF, Rogers G, Lynch G (1994) Facilitation of classical conditioning through enhanced glutamatergic transmission. Neurosci Lett 186:1-4.

Staubli U, Perez Y, Xu F-B, Rogers G, Ingvar M, Stone-Elander S, Lynch G (1994a) Centrally active modulators of glutamate receptors facilitate the induction of long-term potentiation in vivo. Proc Natl Acad Sci USA 91:11158-11162.

Staubli U, Rogers G, Lynch G (1994b) Facilitation of glutamate receptors enhances memory. Proc Natl Acad Sci USA 91:777-781.

Squire LR 1992 Memory and the hippocampus: a synthesis from findings with rats, monkeys, and humans. Psychol Rev 99:195-231.

Vyklicky Jr L, Patneau DK, Mayer ML (1991) Modulation of excitatory synaptic transmission by drugs that reduce desensitization at AMPA/ kainate receptors. Neuron 7:971-984. 